of this paper to the author, who gave the solution in 1945 while a member of the Statistical Research Group of Columbia University, which was working under contract with the National Defense Research Committee of the Office of Scientific Research and Development.

\title{
A COMMENT ON F. N. FRENKIEL'S NOTE “ON THIRD-ORDER CORRELATION AND VORTICITY IN ISOTROPIC TURBULENCE"*
}

\author{
By G. K. BATCHELOR ANd A. A. TOWNSEND (Cambridge University)
}

The short paper by Mr. F. N. Frenkiel [this Quarterly, 6, 86-90 (1948)] calls for some comment. Mr. Frenkiel makes certain assumptions about the correlation functions and derives relations for the decay of energy and mean-square vorticity in isotropic turbulence. These relations are not in agreement with measurements which we have already published. ${ }^{1}$ Mr. Frenkiel concludes that "if account is taken of probable inaccuracies in the experimental data (in Ref. 1), it appears that the agreement (with his deductions) may be satisfactory after all." In answer to this suggestion, we make the following points.

(1) In our opinion there is no possibility that experimental error would account for the discrepancies between our data and Mr. Frenkiel's results. He finds $d \lambda^{2} / d t=7 \nu$, whereas according to our measurements, $d \lambda^{2} / d t=10 \nu$ with a standard deviation of less than $0.4 \nu$. Our measurements were corrected for the effect of finite length of the recording hot-wire but, in any case, the correction leaves $d \lambda^{2} / d t$ unaltered provided the wire length is small enough to be comparable with $\lambda$. Mr. Frenkiel also suggests lack of isotropy as a possible source of error. The presence of isotropy to a sufficient approximation seems to us to have been checked very well by the consistency of measurements of $\bar{u}$ and $\lambda$ (which are related by the energy equations for isotropic turbulence) and of $\overline{u^{2}}, \lambda, f_{0}^{\text {iv }} \lambda^{4}$ and $k_{0}^{\prime \prime \prime} \lambda$ (which are related by the vorticity equation).

(2) There is a strong body of theory ${ }^{2}$ concerning the mechanism of turbulence at high Reynolds numbers, which has been shown ${ }^{3}$ to link up very satisfactorily with the measurements of Ref. 1. Any contrary results, such as that given by Mr. Frenkiel for the variation of $k_{0}^{\prime \prime \prime} \lambda^{3}$ during decay, would therefore need strong experimental and theoretical backing.

(3) Mr. Frenkiel gives no reasons to support his assumption that the correlation curve is self-preserving outside the (small) region in which $r$ is comparable with $\lambda$. Indeed, it leads him into inconsistencies. Later in his paper he assumes, in agreement with our measurements, that $f_{0}^{\mathrm{i} \nu} \lambda^{4}$ is constant during decay. This implies that the correlation curve is also self-preserving for small values of $r$ and it has now been assumed in effect that $f(r)$ is completely self-preserving. Equation (6) of his paper then leads to the conclusion that $\lambda\left(\overline{u^{2}}\right)^{1 / 2} / \nu$ is constant during decay, whereas his published result is that this quantity varies as $t^{-3 / 14}$. Again, he is willing to assume $f_{0}^{\text {iv }} \lambda^{4}$ independent of $t$, and any physical basis for this assumption would seem to lead also to $k_{0}^{\prime \prime \prime} \lambda^{3}$ being independent of $t$ (as our measurements show), but Mr. Frenkiel finds $k_{0}^{\prime \prime \prime} \lambda^{3}$ is proportional to $t^{3 / 14}$.

${ }^{*}$ Received June 16, 1948.

${ }^{1}$ G. K. Batchelor and A. A. Townsend, Proc. Roy. Soc. (A) 190, 534 (1947).

${ }^{2}$ A. N. Kolmogoroff, C. R. Acad. Sci. U.R.S.S. 30, 301 (1941) and 32, 16 (1941).

${ }^{3}$ G. K. Batchelor, Proc. Camb. Phil. Soc. 43, 534 (1947). 